\title{
Repulsion of Resonance States and Exceptional Points
}

\author{
W.D. Heiss \\ Centre for Nonlinear Studies and Department of Physics \\ University of the Witwatersrand, PO Wits 2050, Johannesburg, South Africa
}

PACS: 03.65.Bz, 02.30.Dk, 84.40.-x

Level repulsion is associated with exceptional points which are square root singularities of the energies as functions of a (complex) interaction parameter. This is also valid for resonance state energies. Using this concept it is argued that level anti-crossing (crossing) must imply crossing (anti-crossing) of the corresponding widths of the resonance states. Further, it is shown that an encircling of an exceptional point induces a phase change of one wave function but not of the other. An experimental setup is discussed where this phase behaviour which differs from the one encountered at a diabolic point can be observed.

The dependence on parameters of the energies and widths of resonance states has always been a central focus of interest in virtually all domains of physics. One particular aspect is the repulsion of levels in the complex energy plane. A level repulsion in the complex energy plane can appear as a crossing of, say, their real parts, since the corresponding imaginary parts still can avoid each other; likewise, a genuine repulsion of the real parts can imply a crossing of the imaginary parts. These aspects have been discussed in a variety of contexts: in nuclear and particle physics, for electro-magnetic resonators [1,2] and in results found for absorptive media in solid state physics [3]. An investigation on a more theoretical footing is found in [4].

The purpose of the present paper is twofold. First, we demonstrate that the various types of crossing and/or anti-crossing can be understood from a common principle. They are related to each other by the position of particular singular points of the spectrum, which are called exceptional points (EP) [5]. The second aspect deals with the fact that, if an EP is encircled, the phases of the associated wave functions change in a particular way which is different from the phase behaviour when a genuine degeneracy of levels (a diabolic point) is encircled [6]. At an EP two levels coalesce, but, as is discussed below, an EP is not to be confused with a genuine degeneracy of two resonant states. The fact that there are different types of coalescence of resonance states was pointed out in [4]. However, in the quoted paper, the type of singularity, in fact the concept of an EP was not explicitly employed; rather the effect upon the Green's function or the scattering amplitude was elaborated, which is of lesser interest here. Genuine degeneracies of resonance states have been discussed in the literature, including an associated phase behaviour of the wave functions involved [7, 8 . However, the subject of the present paper which is a generalisation and further expansion of a previous publication [9] addresses a thoroughly different situation.

All essential aspects of exceptional points can be illustrated on an elementary level with a two level model. In fact, for finite or infinite dimensional problems an isolated exceptional point can be described locally by a two dimensional problem [10]. In other words, even though a high or infinite dimensional problem is globally more complex than the two dimensional problem, we do not loose generality for our specific purpose when the restriction to a two dimensional problem is made. For easy illustration we begin with the discussion of

$$
H=\left(\begin{array}{cc}
\epsilon_{1} & 0 \\
0 & \epsilon_{2}
\end{array}\right)+\lambda U\left(\begin{array}{cc}
\omega_{1} & 0 \\
0 & \omega_{2}
\end{array}\right) U^{\dagger}
$$

with

$$
U(\phi)=\left(\begin{array}{cc}
\cos \phi & -\sin \phi \\
\sin \phi & \cos \phi
\end{array}\right) .
$$

This is, up to a similarity transformation, the most general form of a real two dimensional Hamilton matrix of the type $H_{0}+\lambda H_{1}$. We emphasize again that our aim is not in particular directed at a physical model that is describable by a two dimensional problem although there may exist interesting problems in our special context. The example has been chosen for illustration, while the physical application that we have in mind is in general an infinite dimensional situation.

The eigenvalues of $H$ are given by

$$
E_{1,2}(\lambda)=\frac{\epsilon_{1}+\epsilon_{2}+\lambda\left(\omega_{1}+\omega_{2}\right)}{2} \pm R
$$

where

$$
\begin{aligned}
R & =\left\{\left(\frac{\epsilon_{1}-\epsilon_{2}}{2}\right)^{2}\right. \\
& \left.+\left(\frac{\lambda\left(\omega_{1}-\omega_{2}\right)}{2}\right)^{2}+\frac{1}{2} \lambda\left(\epsilon_{1}-\epsilon_{2}\right)\left(\omega_{1}-\omega_{2}\right) \cos 2 \phi\right\}^{1 / 2} .
\end{aligned}
$$

Clearly, when $\phi=0$ the spectrum is given by the two lines

$$
E_{k}^{0}(\lambda)=\epsilon_{k}+\lambda \omega_{k}, \quad k=1,2
$$

which intersect at the point of degeneracy $\lambda=-\left(\epsilon_{1}-\right.$ $\left.\epsilon_{2}\right) /\left(\omega_{1}-\omega_{2}\right)$. When the coupling between the two levels is turned on by switching on $\phi$, the degeneracy is lifted 
and an avoided level crossing occurs. Now the two levels coalesce in the complex $\lambda$-plane where $R$ vanishes. This happens at the complex conjugate points

$$
\lambda_{c}=-\frac{\epsilon_{1}-\epsilon_{2}}{\omega_{1}-\omega_{2}} \exp ( \pm 2 i \phi) .
$$

At these points, the two levels $E_{k}(\lambda)$ are connected by a square root branch point; in fact the two levels are the values of one analytic function on two different Riemann sheets. Obviously, this connection is not of the type encountered at a genuine diabolic point. We stress again that the same nature of singularity prevails also in an $N$-dimensional matrix problem of the type $H_{0}+\lambda H_{1}$.
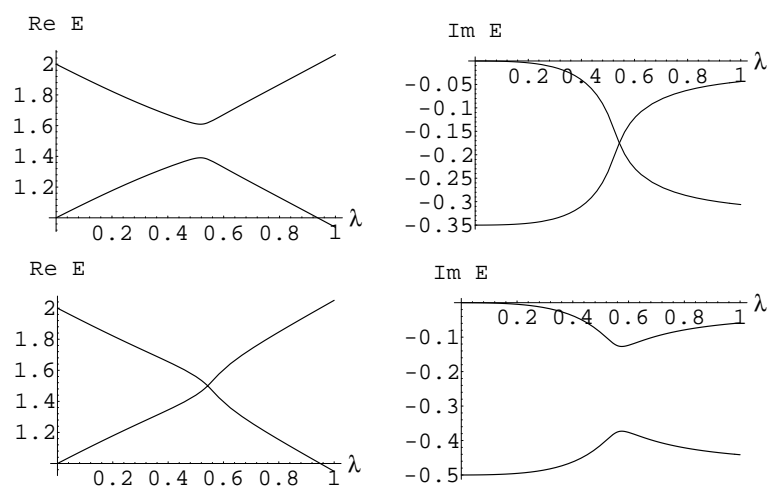

FIG. 1. Level repulsion (width crossing) and level crossing (width repulsion) for smaller (top, $\mu=0.35$ ) and larger (bottom, $\mu=0.5$ ) absorption $\mu$ which is chosen such that the EP lies just below the real $\lambda$-axis in the former and just above in the latter case. The other parameters are $\epsilon_{1}=1, \epsilon_{2}=2, \omega_{1}=1, \omega_{2}=-1, \phi_{1}=0.2$.

The question arises as to whether the existence of the EPs is of physical interest in addition to their pertinent association with level repulsion. Before we turn to actual proposals of encircling an EP in an experiment we first discuss formally the various effects of encircling an EP. Obviously, we we obtain the same information by comparing the results of two different sweeps over appropriate values of $\lambda$, the one by passing an EP on its left hand side and the other on its right hand side. In principle, this can be achieved by choosing complex values of $\lambda$. In order to get closer to an actual experimental situation we expand the Hamiltonian by considering the enlarged model

$$
\begin{aligned}
H=\left(\begin{array}{cc}
\epsilon_{1} & 0 \\
0 & \epsilon_{2}
\end{array}\right) & +\lambda U\left(\phi_{1}\right)\left(\begin{array}{cc}
\omega_{1} & 0 \\
0 & \omega_{2}
\end{array}\right) U^{\dagger}\left(\phi_{1}\right) \\
& -i \mu U\left(\phi_{2}\right)\left(\begin{array}{cc}
\sigma_{1} & 0 \\
0 & \sigma_{2}
\end{array}\right) U^{\dagger}\left(\phi_{2}\right) .
\end{aligned}
$$

The additional term ( $\mu$ real) can be used to describe an absorption while adhering to real values of $\lambda$. Also, the unperturbed energies $\epsilon_{k}$ may be chosen complex, that is including a width. The EP of the enlarged model are situated at

$$
\lambda_{c}=\left(-1+i \mu e^{ \pm 2 i \phi_{2}} \frac{\sigma_{1}-\sigma_{2}}{\epsilon_{1}-\epsilon_{2}}\right) \cdot e^{ \pm 2 i \phi_{1}} \frac{\epsilon_{1}-\epsilon_{2}}{\omega_{1}-\omega_{2}} .
$$

In an experimental situation a judicious choice of these additional parameters can move one of the EP in the $\lambda$-plane close to the real axis. In fact, we now demonstrate that the position of an EP can be arranged in various ways to lie just above or below the real $\lambda$-axis. The different effect of these two situations for the energies, when sweeping over real values of $\lambda$, turns out to be anti-crossing for the real and crossing for the imaginary parts in the one case, and crossing for the real and anti-crossing for the imaginary parts in the other.

In Fig.1 we illustrate the real and imaginary parts of the energies as a function of the real parameter $\lambda$ for two different values of $\mu$. For simplicity, the choice $\phi_{2}=0, \sigma_{1}=1$ and $\sigma_{2}=0$ was made. Other choices lead qualitatively to the same result if $\sigma_{1} \neq \sigma_{2}$. Also, if the unperturbed energies are chosen complex, either in addition or instead of the choice just made, the qualitative picture remains. The switching from level avoidance to level crossing of the real parts, - and associated with it the switching from crossing to avoidance of the related imaginary parts-, is effected by the slipping of the EP over the real $\lambda$-axis. Within the model considered here it can be achieved by starting with different unperturbed widths and/or an absorptive part of the Hamiltonian which couples to the two channels with different strength. In Fig.2 it is demonstrated for a four dimensional model, that our findings are not just the fluke of a two dimensional model [1].
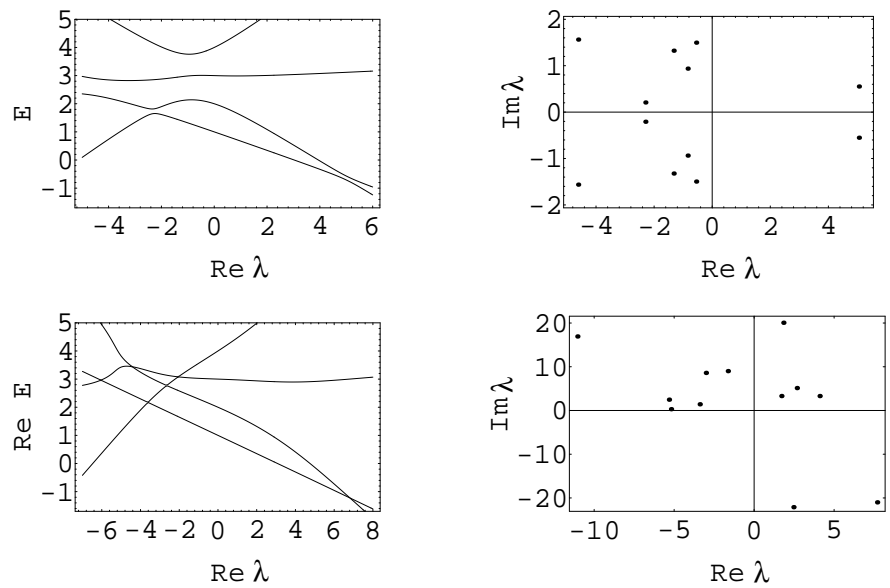

FIG. 2. Effect of strong absorption in an arbitrary four level model. Complex conjugate pairs of EPs of a non-absorptive Hamiltonian (top left) lead to genuine level repulsion (top right). The absorption moves one member of each pair of EPs either into the upper or lower $\lambda$-plane (bottom left) thereby effecting level crossing (bottom right).

The top row shows a usual level repulsion among the four levels together with the exceptional points without absorption, i.e. for a real symmetric Hamiltonian, while in the bottom row the absorption has been made sufficiently strong to enforce the crossing of all levels; the 
last EP which has just slipped over the real axis with increasing absorption lies at $\lambda \approx-5.5$. Note the symmetric positions of the EP with respect to the real axis in the top row which prevails for a selfadjoint Hamiltonian [12]. Also note that the crossing of the real axis by an EP can happen in either direction; this is why, in the particular case considered, two EP are left in the lower half plane for the absorptive Hamiltonian.

We present a topological argument why either the real parts or the imaginary parts must cross when energy trajectories of an absorptive Hamiltonian pass the vicinity of an EP. We denote by $\lambda_{\text {cross }}$ the real part of an EP and follow the trajectories $E_{1,2}(\lambda)$ for real values of $\lambda$ in the interval $\left[\lambda_{\text {cross }}-\delta, \lambda_{\text {cross }}+\delta\right]$. In Fig. 3 the complex numbers $E_{1,2}\left(\lambda_{\text {cross }}-\delta\right)$ are indicated by $A$ and $A^{\prime}$. By definition they have different real and imaginary parts. The endpoints of the trajectories, which are at $E_{1,2}\left(\lambda_{\text {cross }}+\delta\right)$ and denoted by $B$ and $B^{\prime}$, must schematically be situated as indicated, since we consider the vicinity of a square root singularity. Schematically we may assume the singularity to be in the middle of the square like figure. When $\lambda$ is sweeping over the interval $\left[\lambda_{\text {cross }}-\delta, \lambda_{\text {cross }}+\delta\right]$ the energy trajectory starting at $A$ can move to $B$ in which case $A^{\prime}$ must move to $B^{\prime}$ (dotted lines). This is the case of width crossing and level avoidance. The other possibility is that the endpoints are interchanged which is level crossing and width avoidance (dashed lines). Only in the special case where the parameter $\lambda$ moves straight through an EP will both, the real and imaginary part, cross. Since it is a square root singularity the angles between the in and out trajectories at the EP must be at 90 degrees in the energy plane (solid lines).

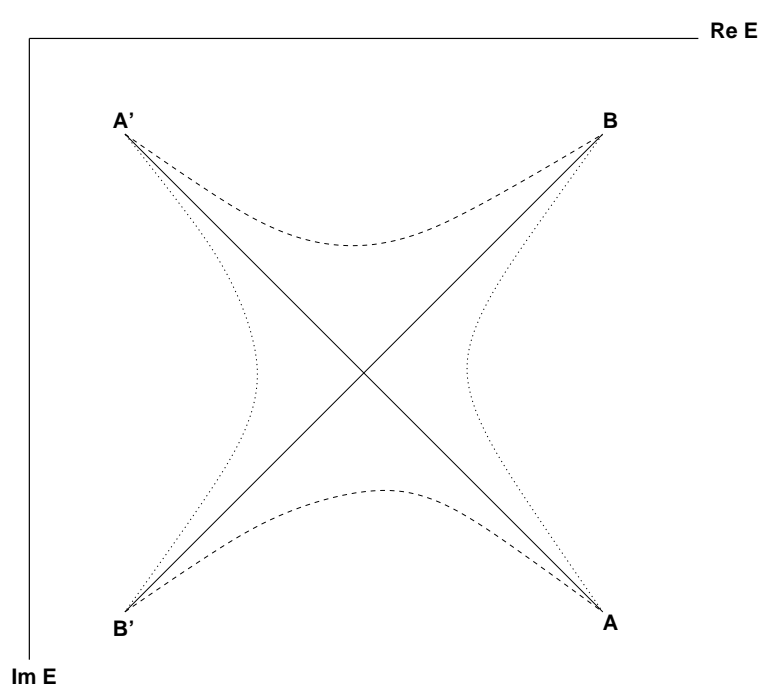

FIG. 3. Schematic energy trajectories in the complex energy plane. Explanations in main text.

The different behaviour of the energy trajectories, depending on a left hand or right hand side passage of an $\mathrm{EP}$, is also reflected in a different behaviour of the corresponding wave functions. It can be intuitively argued, and we confirm this formally below, that we should expect the phase of one but not the other wave function to be different when comparing them behind the point of anti-crossing and crossing of their real parts (Fig.1). In fact, for the situation of level avoidance, it is well known that the wave functions after the point of repulsion are basically as if the levels would have crossed but for a minus sign of one. In the vicinity of the point of repulsion the eigenvectors can be parametrised by an angle $\alpha$ which ranges from 0 to $\pi / 2$ when $\lambda$ is sweeping over the repulsion point. Denoting by $\psi_{1}$ and $\psi_{2}$ the wave functions of the top and bottom level before the repulsion, respectively, we have

$$
\begin{aligned}
& \psi_{\text {top }}=\psi_{1} \cos \alpha-\psi_{2} \sin \alpha \\
& \psi_{\text {bot }}=\psi_{1} \sin \alpha+\psi_{2} \cos \alpha
\end{aligned}
$$

for the wave functions behind the repulsion. In turn, if the levels do cross, the wave functions pertaining to the same levels will not undergo this rotation. As a consequence, behind the crossing or anti-crossing the bottom level is associated with $\psi_{1}$ while the wave function of the top level is $+\psi_{2}$ for the crossing and $-\psi_{2}$ for the anticrossing. This intuitive argument does not really specify which of the two wave functions changes its sign; what matters is the relative change of one of the wave functions. Which one it is in the final comparison depends on the sense of direction by which the EP is encircled.

To confirm more formally the statement about the phases we consider the two situations displayed in Fig.1. We parametrise the state vectors by the complex angle $\theta$, viz.

$$
\psi_{1}(\lambda)=\left(\begin{array}{c}
\cos \theta \\
\sin \theta
\end{array}\right), \quad \psi_{2}(\lambda)=\left(\begin{array}{c}
-\sin \theta \\
\cos \theta
\end{array}\right) .
$$

with

$$
\begin{aligned}
\tan \theta(\lambda) & = \\
( & \left.\lambda\left(\omega_{1}-\omega_{2}\right) \sin 2 \phi_{1}-i \mu\left(\sigma_{1}-\sigma_{2}\right) \sin 2 \phi_{2}\right) / \\
& \left(E_{1}(\lambda)-E_{2}(\lambda)+\epsilon_{1}-\epsilon_{2}+\right. \\
& \left.\lambda\left(\omega_{1}-\omega_{2}\right) \cos 2 \phi_{1}-i \mu\left(\sigma_{1}-\sigma_{2}\right) \cos 2 \phi_{2}\right) .
\end{aligned}
$$

For the value of $\mu$ (and $\phi_{2}=0$ ) which yields the anticrossing we read off from Eq.(10) the expected result: $\theta(0)=0$ and $\theta(\lambda) \rightarrow \phi_{1}$ for $\lambda \gg\left|\left(\epsilon_{1}-\epsilon_{2}\right) /\left(\omega_{1}-\omega_{2}\right)\right|$. In obtaining this result use is made of $E_{1}-E_{2}=2 R \rightarrow$ $\lambda\left(\omega_{1}-\omega_{2}\right)$ for $\lambda \gg\left|\left(\epsilon_{1}-\epsilon_{2}\right) /\left(\omega_{1}-\omega_{2}\right)\right|$. For the other value of $\mu$ yielding the crossing of the levels we now have to observe that we crossed into the other sheet of the square root which means $E_{1}-E_{2}=-2 R \rightarrow-\lambda\left(\omega_{1}-\omega_{2}\right)$. As a consequence we find this time $\tan \theta \rightarrow \tan \left(\phi_{1}+\pi / 2\right)$ which confirms the result. This consideration also clarifies that it is the square root singularity that brings about this particular phase change. The values of $\lambda$ which exceed $\lambda_{\text {cross }}$ get us into different Riemann sheets depending on whether we pass the EP on its right hand or left hand side. 
The different cases as illustrated in Fig.1 have already been experimentally established in an electro-magnetic resonator [13]. Two coupled resonators have been used as experimental setup. The levels of the one have been tuned by a parameter which plays the role of our parameter $\lambda$. As a second parameter, the coupling strength between the resonators has been controllable; we denote this quantity by $x$. For fixed absorption which is achieved by suitable antennas the necessary different widths have been adjusted. The situation has also been modelled by two levels, however without reference to the existence of EPs. With the parametrisation used in the present paper it corresponds to the choice $\mu=0, \phi_{1}=\pi / 4$ and $\epsilon_{k}=E_{k}-i \Gamma_{k} / 2$ with $\Delta \Gamma=\Gamma_{1}-\Gamma_{2} \neq 0$. The exceptional points are then situated at

$$
\lambda_{c}=-\Delta E-i \Delta \Gamma \pm i x
$$

with $\Delta E=\epsilon_{1}-\epsilon_{2}$. In this way, the difference $\Delta \Gamma$ and/or the coupling $x$ can be adjusted such that one EP lies just above or below the real $\lambda$-axis thus giving rise to the two cases illustrated in Fig.1. The equipment used in 13] did not allow a measurement of the phases of the wave functions, i.e. of the field strengths. It appears, however, that this is possible 14].

We stress that exceptional points are a universal phenomenon in contrast to diabolic points. While diabolic points may arise when two real parameters are suitably chosen in a Hamiltonian, exceptional points always occur whenever there is level repulsion. The physically interesting aspect is of course the access to one or more of these points in an experiment. It was demonstrated that this is achievable in dissipative resonators. It is expected that it should be possible in a variety of other systems, systems where interacting resonances prevail. The present paper focusses on level crossing or anticrossing and related phase behaviour for the associated wave functions. Interference effects between the two distinct cases - left hand and right hand passage of an EP - and statistical aspects for large number of resonance states will be the subject of forthcoming considerations.

Acknowledgment The author acknowledges useful discussions with Peter von Brentano as well as the warm hospitality in the Theory Group of the Max Planck Institute at Heidelberg where most part of this paper was written.

[1] P. von Brentano and M. Philipp, Phys.Lett. B454 (1999) 171, and references therein

[2] W.D. Heiss, I. Rotter and M. Müller, Phys.Rev. E58 (1998)2894, and references therein

[3] O. Lefeuvre et.al., App.Phys.Lett. 72 (1998) 856

[4] E. Hernandez and A. Mondragon, Phys.Lett. B326, 1,
(1994); A. Mondragon and E. Hernandez, J. Phys. A26, 5595 (1993)

[5] T. Kato, Perturbation theory of linear operators Springer, Berlin 1966

[6] M.V. Berry, Quantum Chaos, ed. by G. Casati (London: Plenum) 1985; Proc. R. Soc. A239, 45 (1983)

[7] G. Nenciu and G. Rasche, Journal of Physics A (Mathematical and General) 255741 (1992); T.B. Kepler and M.L. Kagan, Phys.Rev.Lett. 66847 (1991); D. Ellinas, S.M. Barnett and M.A. Dupertuis, Phys.Rev. A 393228 (1989)

[8] C. Mimiatura, C. Sire, J. Baudon and J. Bellissard, Eur.Lett. 13, 199 (1990)

[9] W.D. Heiss, Eur.Phys.J. D, in press

[10] W.D. Heiss and W.-H. Steeb, J. Math. Phys. 32, 3003 (1991)

[11] Preliminary simulations in preparation of an experiment clearly confirm the same pattern in an infinite dimensional problem: C. Dembowski, Technische Universität Darmstadt, private communication.

[12] W.D. Heiss and A.L. Sannino, J. Phys. A23 1167 (1990); W.D. Heiss and A.L. Sannino, Phys. Rev. A43 4159 (1991)

[13] M. Philipp, Doctoral Thesis, University of Köln, 1997

[14] Achim Richter, Technische Universität Darmstadt, private communication. 